Apidologie, 1987, 18 (3), 253-266

\title{
ÉTUDE THÉORIQUE SUR LA SÉLECTION DU CARACTÈRE “PRODUCTION DE MIEL " CHEZ L'ABEILLE II. PLAN DE SÉLECTION COMBINÉE DE REINES EN FÉCONDATION NATURELLE
}

\author{
J.M. CORNUET \\ I.N.R.A., Station de Zoologie et d'Apidologie, 84140 Montfavet \\ C. CHEVALET \\ Laboratoire de Génétique Cellulaire, \\ B.P. 27, 31326 Castanet Tolosan Cedex
}

\begin{abstract}
RÉSUMÉ
Cet article présente un plan de sćlection qui ne fait appel qu'à l'élevage de reines et à la mesure individuelle du rendement en miel des ruches. Le choix des colonies repose sur le calcul d'un indice de sćlcction combinant leur propre valeur et celle de colonies qui leur sont apparentées. La discussion porte sur le nombre de colonies sélectionnées qui rend maximum le progrès génétique tout en assurant le maintien de la consanguinité en dessous d'un seuil donné et sur le gain relatif de cette méthode par rapport à la sćlection massale.
\end{abstract}

\section{INTRODUCTION}

Dans la première partie de cette étude, nous avons présenté un modèle génétique et statistique pour analyser le rendement en miel de colonies apparentées (Chevalet et Connuet, 1982 a). Ensuite deux plans de croisements ont été proposés, l'étude se bornant à la construction des indices de sélection. Dans cette deuxième partie, nous analysons plus en détail le premier de ces deux plans, car il présente à nos yeux un intérêt pour les apiculteurs désireux d'améliorer leur cheptel. Il se trouve en effet à la portée de beaucoup d'entre eux, puisqu'il ne fait intervenir que la mesure individuelle du rendement en miel des ruches et la maîtrise de l'élevage des reines.

Son principe est le suivant : à chaque génération, plusieurs colonies sont sélectionnées sur la base de leur rendement et de celui de colonies qui leur sont apparentées. A partir des colonies sélectionnées, seront élevées les reines de la génération suivante qui seront fécondées naturellement. Toutes les 
dispositions seront prises pour favoriser la fécondation de ces reines par des mâles issus des mêmes colonies sélectionnées.

Après avoir donné une description plus complète de ce plan, nous considèrerons les paramètres influençant la réponse à la sélection, puis ceux concernant l'évolution de la consanguinité. Nous comparerons son efficacité théorique à celle de la sélection massale. Enfin, compte tenu de l'absence de contrôle des accouplements, nous tenterons d'analyser l'effet de la fécondation par un mélange de mâles (sélectionnés et non sélectionnés).

\section{DESCRIPTION DU PLAN DE SÉLECTION}

Pour clarifier cette présentation, nous exposerons séparément l'organisation des opérations dans le temps et dans l'espace.

\section{I.1. Calendrier des opérations}

L'expérience débute l'année $(0)$.

Au printemps (0), un élevage de reines est effectué à partir de $\mathrm{R}$ colonies non apparentées. Ces $\mathrm{R}$ colonies peuvent être choisies au hasard, mais le sélectionneur a naturellement intérêt à choisir parmi ses meilleures colonies. Les jeunes reines sont ensuite fécondées naturellement et installées dans des colonies. A la fin de cette étape, on doit disposer de $\mathrm{S}$ colonies filles par souches, soit un total de RS colonies dont on testera le rendement en miel.

A l'automne (0), le poids de ces RS colonies est enregistré individuellement ainsi que celui du nourrissement éventuel effectué pour égaliser leurs chances de survie au seuil de l'hiver.

Au printemps (1), on note à nouveau les nourrissements éventuels.

A l'automne (1), les rendements en miel de chaque colonie sont calculés selon la formule de Fresnaye et al. (1974) :

$\mathrm{H} .=\mathrm{H}_{1}+\mathrm{H}_{2}+\ldots+\mathrm{K}_{1}-\mathrm{K}_{0}-\mathrm{N}$

où $H_{1}, H_{2}, \ldots$ sont les récoltes de l'année (1),

$\mathrm{K}_{\mathrm{i}}$ est le poids de la ruche avant l'hivernage (année i),

$\mathrm{N}$ est le poids total de sucre de nourrissement.

Pendant l'hiver (2), les indices de sélection des reines survivantes sont calculés, ce qui permet de les classer de la meilleure à la moins bonne en trois groupes :

- de la $1^{\text {re }}$ à la $P^{\text {eme }}:$ groupe 1 ,

- de la $1^{\text {re }}$ à la $R^{\text {eme }}$ : groupe 2 ,

- de la $(\mathrm{R}+1)^{\text {eme }}$ à la dernière : groupe 3 . 
Le groupe 1 est constitué par les colonies dans lesquelles seront élevés les mâles. En général, ce groupe est inclus dans le groupe $2(P<R)$.

Au printemps (2), les $\mathrm{R}$ colonies dont les reines appartiennent au groupe 2 constituent les souches sur lesquelles sont élevées les RS reines de la génération suivante. Les jeunes reines sont fécondées naturellement par des mâles provenant : rons,

- dans une proportion $1-\beta$, des colonies non sélectionnées des envi-

- dans une proportion $B$, des reines du groupe 1 qui auront été poussées à produire des mâles.

Les colonies, dont les reines appartiennent au groupe 3, servent à l'élevage des jeunes reines et à la constitution des nuclei de fécondation. Enfin toutes les colonies (groupes 2 et 3) sont utilisées pour former les essaims des RS colonies de la nouvelle génération.

A partir de ce moment, les mêmes opérations seront effectuées tous les deux ans de façon identique pendant toute la durée d'application du plan. Ces deux années représentent donc l'intervalle de génération.

\subsection{Organisation spatiale}

Les différentes opérations peuvent se résumer en trois points : élevage des reines, fécondation de ces reines et testage des colonies. Pour les deux derniers points, se pose la question de l'emplacement. Puisque les jeunes reines doivent être fécondées en partie par des mâles issus de colonies sélectionnées, ces dernières doivent être à proximité des nuclei de fécondation et de préférence dans le même rucher (rucher de fécondation). L'isolement relatif de ce rucher et la densité des colonies non sélectionnées dans le voisinage influeront sur la valeur du paramètre $\beta$.

Ensuite, il faut considérer les conditions dans lesquelles les colonies effectueront leurs récoltes. Si toutes les ruches peuvent rester groupées dans un même rucher (ou une même suite de ruchers en cas de transhumance), il suffira de veiller à éviter au maximum la dérive. Par contre, si l'apiculteur est obligé de séparer son cheptel en plusieurs groupes, il y aura lieu de prendre des précautions supplémentaires pour ne pas biaiser les résultats. D'une part, chaque famille de colonies sœurs sera répartie le plus également possible entre les différents groupes. D'autre part, dans le calcul des indices, il sera tenu compte des effets communs au groupe.

Il reste à évoquer la question des témoins dont la présence est nécessaire pour estimer le progrès effectivement réalisé. Ces colonies témoins seront disposées dans les mêmes ruchers que les colonies soumises à la sélection et 
devront avoir à leur tête des reines de même âge. Par contre, ces reines élevées à partir de reines tirées au hasard dans la population témoin devront être fécondées naturellement par des mâles non issus de la population sélectionnées. Ceci implique la création d'un deuxième rucher de fécondation hors de portée des mâles de la population sélectionnée.

Jusqu'à présent, aucune indication sur la taille du schéma, à savoir les valeurs de $R, S$ et $P$, n'a été fournie. En fait, pour un nombre total de ruches (RS), il faut choisir les valeurs de ces paramètres qui procurent la meilleure réponse théorique, tout en maintenant la consanguinité à un taux acceptable.

\section{RÉPONSE THÉORIQUE À LA SÉLECTION}

Dans tous ce qui suit, nous avons repris les notations et certains résultats de la première partie (Chevalet et Cornuet, $1982 \mathrm{a}$ ). Pour simplifier la lecture, nous les rappellerons brièvement.

$h_{i}^{\prime \prime}(1 \leqslant i \leqslant R)$ est la performance de la i-ème colonie mère.

$\mathrm{h}_{\mathrm{ij}}^{\prime}(1 \leqslant \mathrm{j} \leqslant \mathrm{S})$ est la performance de la $\mathrm{j}$-ème colonie fille dont la reine est issue de la i-ème colonie mère.

$h_{i j}$ est la performance d'une colonie dont la reine, issue de la colonie (i, j), a été fécondée par des mâles aléatoires.

$h_{\mathrm{i}}^{\prime}$ représente la moyenne des $\mathrm{S}$ colonies-filles de la i-ème famille.

$\mathrm{h}_{\mathrm{i} .}^{\prime}=\frac{1}{\mathrm{~S}} \sum_{\mathrm{i}=1}^{\mathrm{S}} \mathbf{h}_{\mathrm{ii}}^{\prime}$

Toutes ces performances sont supposées corrigées pour les effets systématiques (rucher, série d'élevage, colonie éleveuse, etc.).

$J_{i j}$ est un indice de sélection qui sert à estimer la valeur de $h_{i j}$. En fait, selon l'information disponible, nous envisagerons trois indices possibles :

JA sur la valeur individuelle de la colonie,

JB sur la valeur individuelle combinée avec la moyenne de ses sœurs,

JC sur une combinaison incluant la valeur de la colonie, la moyenne de sa famille et la valeur de la colonie mère.

Ces indices s'écrivent :

$$
\mathbf{J A}_{\mathrm{ij}}=\mathbf{A}_{2} \mathbf{h}_{\mathrm{ij}}^{\prime}
$$

$$
\begin{aligned}
& J B_{i j}=\frac{A_{2}-A_{3}}{Y-C}\left(h_{i j}^{\prime}-h_{i .}^{\prime}\right)+\frac{A_{4}}{Z} h_{i .}^{\prime} \\
& J C_{i j}=\frac{A_{2}-A_{3}}{Y-C}\left(h_{i j}^{\prime}-h_{i .}^{\prime}\right)+\frac{A_{4}}{Z} h_{i .}^{\prime}+\frac{Z A_{1}-T A_{4}}{Z X-T^{2}}\left(h_{i}^{\prime \prime}-\frac{T}{Z} h_{i}^{\prime}\right)
\end{aligned}
$$




$$
\begin{array}{ll}
\text { avec } & X=\operatorname{var}\left(h_{i}^{\prime \prime}\right) \\
Y & =\operatorname{var}\left(h_{i j}^{\prime}\right) \\
C & =\operatorname{cov}\left(h_{i j}^{\prime}, h_{i k}^{\prime}\right)(j \neq k) \\
Z & =\dot{Y} / S+(S-1) C / S \\
T & =\operatorname{cov}\left(h_{i}^{\prime \prime}, h_{i j}^{\prime}\right) \\
A_{1} & =\operatorname{cov}\left(h_{i}^{\prime \prime}, h_{i j}\right) \\
A_{2} & =\operatorname{cov}\left(h_{i j}^{\prime}, h_{i j}\right) \\
& A_{3}=\operatorname{cov}\left(h_{i k}^{\prime}, h_{i j}\right) \text { la reine ik étant la tante de la reine ij } \\
\text { et } \quad A_{4}=A_{2} / S+(S-1) A_{3} / S
\end{array}
$$

En supposant que les observations sont normées $(\mathrm{X}=\mathrm{Y}=1)$ et que la covariance mère fille ne change pas d'une génération à la suivante $\left(T=A_{2}\right)$ et en posant $\mathrm{W}=\mathrm{A}_{4} / \mathrm{T}$, les indices s'écrivent encore :

$$
\begin{aligned}
\mathrm{JA}_{\mathrm{ij}} & =\mathrm{T} \mathbf{h}_{\mathrm{ij}}^{\prime} \\
\text { (1b) } \mathrm{JB}_{\mathrm{ij}} & =\frac{\mathrm{T}(1-\mathrm{W})}{1-\mathrm{Z}}\left(\mathbf{h}_{\mathrm{ij}}^{\prime}-\mathbf{h}_{\mathrm{i}}^{\prime}\right)+\frac{\mathrm{WT}}{\mathrm{Z}} \mathbf{h}_{\mathrm{i}}^{\prime} \\
\mathrm{JC}_{\mathrm{ij}} & =\frac{\mathrm{T}(1-\mathrm{W})}{1-\mathrm{Z}}\left(\mathbf{h}_{\mathrm{ij}}^{\prime}-\mathbf{h}_{\mathrm{i}}^{\prime}\right)+\frac{\mathrm{WT}}{\mathrm{Z}} \mathbf{h}_{\mathrm{i} .}^{\prime}+\frac{\mathrm{ZA}_{1}-\mathrm{WT}^{2}}{\mathrm{Z}-\mathrm{T}^{2}}\left(\mathbf{h}_{\mathrm{i}}^{\prime \prime}-\frac{\mathrm{T}}{\mathrm{Z}} \mathbf{h}_{\mathrm{i}}^{\prime}\right)
\end{aligned}
$$

A la première génération, seuls les paramètres $\mathrm{Y}, \mathrm{C}$ et $\mathrm{Z}$ sont estimables. Les indices $\mathrm{JB}$ et $\mathrm{JC}$ ne peuvent alors s'écrire qu'au prix d'hypothèses assez gratuites telles que par exemple $\mathrm{T}=2 \mathrm{C}$ et $\mathrm{A}_{3}=\mathrm{C} / 2$. Il paraît tout autant valable de sélectionner les meilleures colonies de cette génération (sélection individuelle correspondant à l'indice JA).

A la deuxième génération, tous les paramètres sont estimables, sauf la covariance $A_{1}$ entre colonie grand-mère et colonie petite-fille. Les formules (9) et (10) de la première partie (op. cit.) indiquent que cette covariance peut-être évaluée à partir de la covariance $\mathrm{T}$. Pour cela, il faut supposer que, dans l'expression théorique de $\mathrm{T}$, on peut négliger le terme correspondant à la covariance des effets de dominance des contributions de la reine et des ouvrières au caractère. La discussion de cette hypothèse (op. cit.) a montré qu'elle était en général légitime. A partir de la troisième génération, il devient possible d'estimer directement la covariance $\mathrm{A}_{1}$; néanmoins, celle-ci ne peut être estimée qu'à partir de l'échantillon des colonies grand-mères des colonies de cette génération, qui peuvent être peu nombreuses, de sorte que l'estimation, sera très peu précise. Il en résulte que l'évaluation de $A_{1}$ à partir de $T$ demeure préférable. On peut tout aussi bien se limiter à l'indice JB. En effet, sur des exemples numériques, il est apparu que la prise en compte de la 
performance de la colonie-mère n'apportait le plus souvent au progrès génétique qu'un gain imperceptible. Dans ces cas-là, l'utilisation de l'indice JB présente l'avantage d'écarter un paramètre estimé de façon trop imprécise.

Le progrès génétique attendu de cette sélection peut être décomposé, à chaque génération, en progrès attendu par la voie femelle, et progrès indirect par la voie mâle. Le premier est donné directement par le produit de l'intensité de sélection par l'écart-type de l'index $\mathbf{J}$ :

$$
\Delta \mathrm{G}_{\varphi}=i \sigma_{\mathrm{j}}
$$

L'intensité de sélection $i$ est fonction de la proportion 1/S des reines sélectionnées, du nombre $\mathrm{R}$ de familles, et de la corrélation $\mathrm{t}$ entre les indices de colonies sœurs. Hill (1976) a donné une expression approchée de cette quantité :

$$
i(\mathrm{~S}, \mathrm{R}, \mathrm{t})=i_{\mathrm{o}}(\mathrm{S})-\frac{\mathrm{S}-1}{2 i_{\mathrm{o}}(\mathrm{S})(\mathrm{RS}-\mathrm{RSt}+\mathrm{Rt}+1)}
$$

où $i_{0}(\mathrm{~S})$ est l'intensité de sélection réalisée dans une population infinie quand on garde la même proportion $1 / \mathrm{S}$ des meilleurs individus. Sмrтн (1969) a donné une forme approchée de cette fonction :

$$
i_{\mathrm{o}}(\mathrm{S})=0,8+0,41 \ln (\mathrm{S}-1)
$$

Les variances et les corrélations des indices sont ici, respectivement :

$$
\begin{aligned}
& \sigma_{\mathrm{JA}}^{2}=\mathrm{T}^{2} \\
& \mathrm{t}_{\mathrm{jA}}=\mathrm{C} \\
& \sigma_{I B}^{2}=T^{2}\left[1+\frac{(Z-W)^{2}}{Z(1-Z)}\right] \\
& \text { (2) } t_{\mathrm{sB}}=\frac{\frac{(1-W)^{2}}{(1-Z)(1-S)}+\frac{W^{2}}{Z}}{1+\frac{(Z-W)^{2}}{Z(1-Z)}} \\
& \sigma_{\Im C}^{2}=T^{2}\left[1+\frac{(\mathrm{Z}-\mathrm{W})^{2}}{\mathrm{Z}(1-\mathrm{Z})}+\frac{\left(\mathrm{ZA}_{1}-\mathrm{WT}^{2}\right)^{2}}{\mathrm{ZT}^{2}\left(\mathrm{Z}-\mathrm{T}^{2}\right)}\right] \\
& \mathrm{t}_{\mathrm{c}}=\frac{\frac{(1-W)^{2}}{(1-\mathrm{Z})(1-\mathrm{S})}+\frac{\mathrm{W}^{2}}{\mathrm{Z}}+\frac{\left(\mathrm{ZA}_{1}-\mathrm{WT}^{2}\right)^{2}}{\mathrm{ZT}^{2}\left(\mathrm{Z}-\mathrm{T}^{2}\right)}}{1+\frac{(\mathrm{Z}-\mathrm{W})^{2}}{\mathrm{Z}(1-\mathrm{Z})}+\frac{\left(\mathrm{ZA}_{1}-\mathrm{WT}^{2}\right)^{2}}{\mathrm{ZT}^{2}\left(\mathrm{Z}-\mathrm{T}^{2}\right)}}
\end{aligned}
$$


La réponse indirecte apportée par la voie mâle est proportionnelle :

- à la fraction $B$ de mâles sélectionnés dans l'ensemble des mâles fécondant les reines,

- à l'intensité de sélection $j$ correspondant à la fraction P/RS de reines sélectionnées pour produire des mâles,

- au coefficient, $b_{W / J}$, de la régression sur l'indice de sélection $J$, de la contribution $\mathrm{W}$ des ouvrières à la performance d'une colonie dont la reine a été fécondée par des mâles issus d'une reine sélectionnée (première partie, II$5)$.

La réponse indirecte par les mâles s'écrit alors :

$$
\Delta \mathrm{G}_{\sigma^{\prime}}=\beta j \mathrm{~b}_{\mathrm{w}, \mathrm{s}} \sigma_{\mathrm{s}}
$$

\section{RÉSULTATS ET DISCUSSION}

\section{III.1. Optimisation des effectifs du plan}

Si l'on fixe la valeur des composantes génétiques de la variance, la réponse à la sélection est une fonction croissante de $S$ (plus $S$ est grand et plus la sélection est sévère) et de $\mathrm{R}$ (la dérivée partielle de $\mathrm{i}$ par rapport à $\mathrm{R}$ est positive). En pratique, le nombre total RS de ruches est imposé par les possibilités matérielles de l'exploitation. C'est pourquoi, les valeurs des deux paramètres $\mathrm{R}$ et $\mathrm{S}$ seront choisies de telle sorte que :

- le progrès génétique soit maximum,

- la consanguinité soit maintenue à un taux acceptable,

- ces deux paramètres soient en accord avec l'effectif total du plan.

La consanguinité, qui est un facteur très néfaste pour la colonie d'abeilles, augmente lorque le nombre de parents, $\mathrm{R}$, diminue. Dans un travail antérieur (Chevalet et Cornuet, $1982 \mathrm{~b}$ ), nous avons montré qu'avec un coefficient $\beta$ égal à 0,75 , il suffit de prendre 10 reines pour l'élevage des reines $(R=10)$ et entre 5 et 10 reines pour l'élevage des mâles $(5 \leqslant P \leqslant 10)$ pour que la consanguinité moyenne de la population ne dépasse jamais 0,1 .

Le progrès génétique est une fonction complexe de $\mathrm{S}: \mathrm{S}$ intervient à la fois dans l'intensité de sélection (directement et à travers la corrélation $t$ pour les sélections combinées), dans l'écart-type de l'indice et dans le coefficient $b_{\mathrm{W} / \mathrm{J}}$ (sélections combinées). Nous avons donc été conduits à effectuer la recherche des valeurs optimales de $\mathrm{S}$ et de $\mathrm{R}$ par ordinateur. Les conditions envisagées ont été les suivantes :

1) L'effectif total (RS) a pris les valeurs suivantes : $30,60,120,240,480$ et 960 . 
2) Pour chaque effectif, $\mathrm{S}$ a pris toutes les valeurs entières entre 2 et RS/ 10 , ou, ce qui revient au même, $\mathrm{R}$ a varié de 10 à $\mathrm{RS} / 2$.

3) Les six variances génétiques définies dans la première partie $\left(V_{A}^{q}, V_{A}^{q w}\right.$, $V_{A}^{w}, V_{D}^{d}, V_{D}^{\text {gw }}$ et $\left.V_{D}^{w}\right)$ ont varié de la façon suivante :

- la somme des variances additives et la somme des variances de dominance ont pris respectivement les valeurs suivantes : $0,1,0,2$ et 0,3 ( 9 combinaisons),

- pour chaque combinaison, les composantes "reine", "reineouvrières" et "ouvrières" ont représenté soit la moitié soit le quart de la variance correspondante ( 3 combinaisons par valeur de la variance).

4) Le coefficient $B$ a pris les valeurs 1 et 0 .

5) Nous avons considéré un nombre efficace de mâles ayant fécondé une reine égal à 6 , ce qui conduit aux valeurs des coefficients de parenté et d'identité $\bar{\phi}=1 / 6$ et $\mathrm{d}=1 / 12$.

6) L'intensité de sélection a été prise identique pour les deux voies mâle et femelles $(P=R)$.

Les valeurs de $\mathrm{T}, \mathrm{W}$ et $\mathrm{Z}$ se calculent aisément à partir des valeurs des composantes de la variance génétique [formules (9) et (10) de la première partie]. On en déduit donc pour chaque type de sélection la valeur de l'écarttype de l'indice et de la corrélation intraclasse $t$ [formules (2)], ce qui permet de calculer l'intensité de sélection. Le coefficient de régression s'estime lui aussi à partir des mêmes paramètres. Selon le type de sélection envisagé, il est égal à :

$$
\begin{aligned}
& \mathrm{b}_{\mathrm{W} / \mathrm{A}}=\frac{1}{\mathrm{~T}}\left(\mathrm{~V}_{\mathrm{A}}^{\mathrm{qW}} / 2+\mathrm{V}_{\mathrm{A}}^{\mathrm{w}} / 4\right) \\
& \mathrm{b}_{\mathrm{W} / \mathrm{B}}=\frac{1}{\operatorname{var}(\mathrm{JB})}\left(\mathrm{V}_{\mathrm{A}}^{\mathrm{qW} / 2}+\mathrm{V}_{\mathrm{A}}^{\mathrm{w}} / 4\right)\left[\frac{\mathrm{T}(1-\mathrm{W})}{1-\mathrm{Z}}+\frac{\mathrm{T}(\mathrm{W}-\mathrm{Z})}{\mathrm{Z}(1-\mathrm{Z})}(1 / \mathrm{S}+(\mathrm{S}-1) \bar{\phi} / \mathrm{S})\right] \\
& b_{w / J C}=\frac{1}{\operatorname{var}(J C)}\left[( \frac { V _ { A } ^ { q w } } { 2 } + \frac { V _ { A } ^ { w } } { 4 } ) \left\{\frac{T(1-W)}{1-Z}\right.\right. \\
& \left.+\left(\frac{\mathrm{T}(\mathrm{W}-\mathrm{Z})}{\mathrm{Z}(1-\mathrm{Z})}-\frac{\mathrm{T}\left(\mathrm{ZA}_{1}-\mathrm{WT}^{2}\right)}{\mathrm{Z}\left(\mathrm{Z}-\mathrm{T}^{2}\right)}\right)(1 / \mathrm{S}+(\mathrm{S}-1) \bar{\phi} / \mathrm{S})\right\} \\
& \left.+\left(\frac{V_{A}^{\text {qw }}}{2}+\bar{\phi} V_{A}^{w}\right) \frac{Z A_{1}-W T^{2}}{Z-T^{2}}\right]
\end{aligned}
$$


Il ressort de cette étude que dans toutes les combinaisons étudiées, le progrès génétique est maximum pour la valeur minimale de $R(R=10)$, quelle que soit la valeur de $S$.

\section{III.2. Comparaison des types de sélection}

Les formules (2) indiquent clairement la croissance de l'écart-type de l'indice lorsque l'on passe de la sélection massale à la sélection combinée 1 (valeur individuelle + moyenne de la famille), puis à la sélection combinée 2 (valeur individuelle + moyenne de la famille + valeur de la colonie mère). Il s'agit là d'un résultat classique (Lush, 1947). Pour ce qui est de la corrélation intraclasse des indices de sélection, la valeur est nécessairement plus élevée en sélection combinée qu'en sélection massale puisque l'indice en sélection combinée prend en compte la moyenne familiale, commune à toutes les colonies de la famille. Il en résulte que l'intensité de sélection, qui est une fonction décroissante de cette corrélation, est inférieure en sélection combinée par rapport à la sélection massale. Toutefois, dans l'ensemble des situations considérées au paragraphe précédent, l'efficacité théorique de la sélection croît en passant de la sélection massale à la sélection combinée 1, puis à la sélection combinée 2. De plus, en se limitant aux situations ci-dessus, plusieurs observations sont possibles.

D'abord, l'augmentation relative du progrès génétique par rapport à la sélection massale varie entre $0,6 \%$ et $67,5 \%$ pour la sélection combinée 1 et entre $5,1 \%$ et $68,1 \%$ pour la sélection combinée 2 . Pour les deux types de sélection combinée, cette augmentation relative du progrès est une fonction croissante de S.

Ensuite, la différence d'efficacité entre les deux sélections combinées décroît quand $S$ augmente.

Pour les trois types de sélection, le progrès est supérieur pour les fortes valeurs des variances génétiques (variances additives et variances de dominance). L'efficacité relative des sélections combinées est alors minimale. Au contraire, elle est maximale pour les faibles valeurs des variances génétiques.

Enfin, l'augmentation relative du progrès en sélection combinée diminue quand $B$ augmente.

Il ressort de ces calculs que la sélection combinée est d'autant plus intéressante que la taille des familles est élevée, que les variances génétiques sont faibles et que la population est ouverte au niveau des mâles. Par contre, la sélection massale, plus simple, semble le meilleur choix lorsque les effectifs familiaux sont faibles et que les variances génétiques sont relativement fortes. Les imprécisions d'estimation des paramètres génétiques, liées aux effectifs du plan de sélection, viennent renforcer ces conclusions (Sales et HiLl, 1976). 


\section{II.3. Influence de l'ouverture de la population sur le progrès à long terme}

Jusqu'ici, les calculs de progrès génétique n'ont porté que sur la première génération. Si la population est ouverte $(B<1)$, le progrès génétique est freiné par l'apport de gènes en provenance de mâles non sélectionnés. D'un autre côté, par l'intermédiaire des mâles sélectionnés, la sélection profite aux colonies extérieures au plan mais situées au voisinage du rucher de fécondation. Il est possible de modéliser les effets de la sélection sur le progrès génétique des deux populations (sélectionnée et non-sélectionnée). Comme les échanges se font par l'intermédiaire des mâles, les variations du progrès génétiques induits par ces échanges seront proportionnelles :

- à la fraction de mâles en provenance de l'autre population,

- à la différence de niveau génétique entre les deux populations,

- au coefficient de régression de la contribution d'ouvrières à la performance de leur colonie sur la performance des colonies dont sont issus leurs pères.

Si l'on note $\mathrm{H}_{\mathrm{g}}$ le niveau moyen de la population sélectionnée à la génération $\mathrm{g}$ et $\mathrm{K}_{\mathrm{g}}$ celui de la population environnante, nous pouvons écrire :

$$
\begin{gathered}
\mathrm{H}_{\mathrm{g}+1}-\mathrm{H}_{\mathrm{g}}=i \sigma_{\mathrm{J}}+\beta j \sigma_{\mathrm{J}} \mathrm{b}_{\mathrm{w} / \mathrm{J}}+(1-\beta) \mathrm{b}^{\prime}\left(\mathrm{K}_{\mathrm{g}}-\mathrm{H}_{\mathrm{g}}\right) \\
\mathrm{K}_{\mathrm{g}+1}-\mathrm{K}_{\mathrm{g}}=\beta^{\prime} \mathrm{b}^{\prime}\left(\mathrm{H}_{\mathrm{g}}-\mathrm{K}_{\mathrm{g}}\right)
\end{gathered}
$$

où $B^{\prime}$ représente la proportion de mâles provenant de la population sélectionnée et participant à la fécondation des reines de la population environnante et $b^{\prime}$ est le coefficient présenté ci-dessus.

En supposant les paramètres génétiques constant d'une génération à la suivante, l'évolution du niveau moyen des deux populations en fonction de la génération est de la forme :

$$
\begin{aligned}
\mathrm{H}_{\mathrm{g}}= & \frac{\beta^{\prime}}{1-\beta+\beta^{\prime}} \mathrm{g} \Delta \mathrm{G}+\frac{1-\beta}{\left(1-\beta+\beta^{\prime}\right)^{2} \mathrm{~b}^{\prime}} \Delta \mathrm{G}\left(1-\left\{1-\left(1-\beta+\beta^{\prime}\right) \mathrm{b}^{\prime}\right\}^{\mathrm{g}}\right) \\
\mathrm{K}_{\mathrm{g}}= & \frac{\beta^{\prime}}{1-\beta+\beta^{\prime}} \mathrm{g} \Delta \mathrm{G}-\frac{\beta^{\prime}}{\left(1-\beta+\beta^{\prime}\right)^{2} \mathrm{~b}^{\prime}} \Delta \mathrm{G}\left(1-\left\{1-\left(1-\beta+\beta^{\prime}\right) \mathrm{b}^{\prime}\right\}^{\mathrm{g}}\right) \\
& \text { où } \Delta \mathrm{G}=i \sigma_{\mathrm{s}}+\beta j \sigma_{\mathrm{J}} \mathrm{b}_{\mathrm{w} / \mathrm{I}}
\end{aligned}
$$

Les figures 1 et 2 illustrent cette évolution pour deux combinaisons différentes des paramètres génétiques correspondant à des héritabilités respectives de 0,075 et 0,3 (cf. conclusion de la première partie) et pour 3 valeurs de $B$. Pour ces calculs, nous avons supposé que le coefficient $B^{\prime}$ moyen sur une surface circulaire entourant le rucher de fécondation était égal au tiers du coefficient $\beta$. Cela revient à admettre que le coefficient $\beta^{\prime}$ varie linéairement depuis le centre du cercle (où il est égal à $B$ ) jusqu'au périmètre (où il devient 


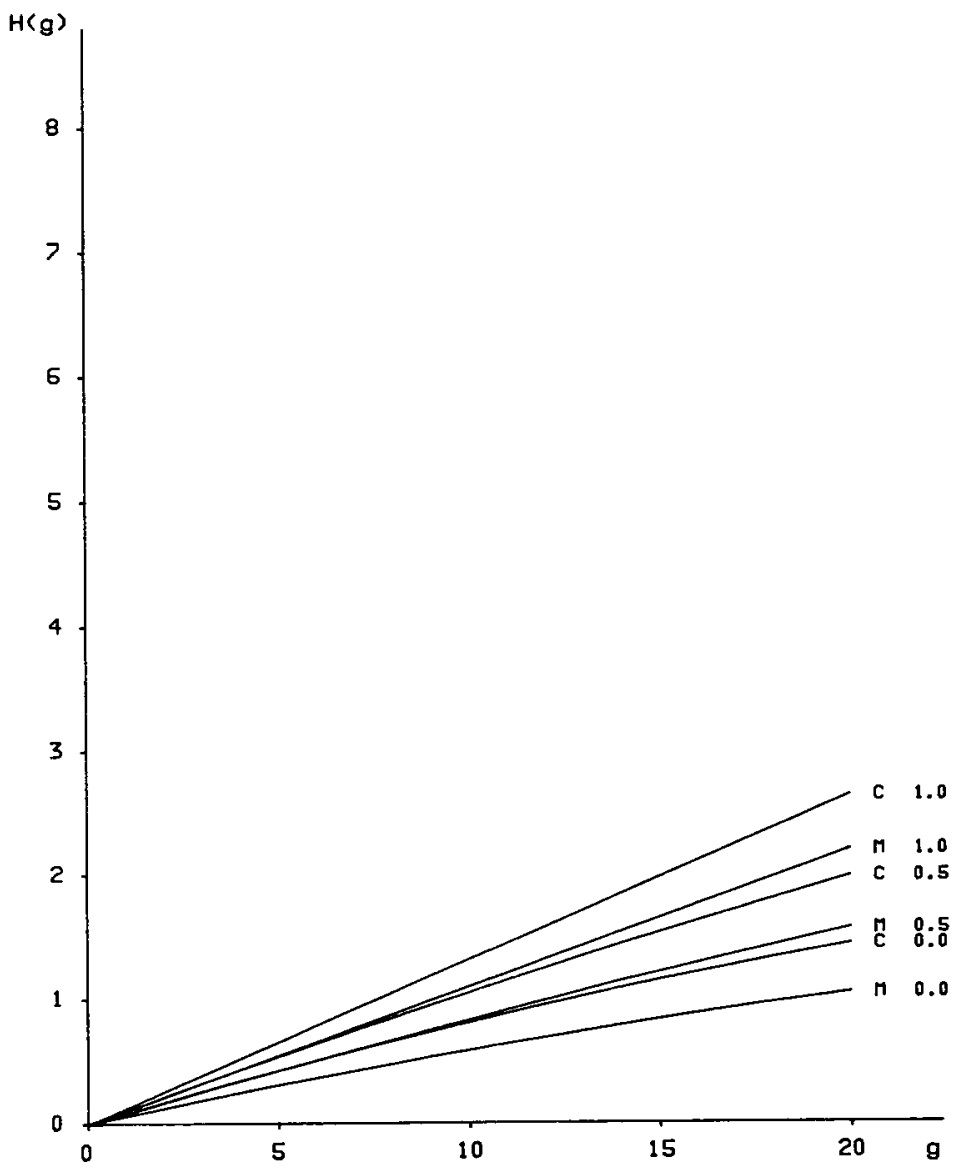

FIG. 1. - Evolution théorique du niveau génétique d'une population sélectionnée (unité : écart-type phénotypique) en fonction des générations.

Courbes $M$ : sélection massale (indice JA).

Courbes $\mathrm{C}$ : sélection combinée (indice JB).

La valeur indiquée après la lettre est celle du paramètre $B$ (proportion de mâles en provenance des colonies sélectionnées).

Les autres paramètres ont les valeurs suivantes :

$$
R=10, S=12, V_{A}=V_{A}^{q^{w}}=V_{D} g^{w}=V_{D}^{w}=0,025, V_{A}^{w}=V_{D}^{q}=0,050
$$

FIG. 1. - Theoretical change of the genetic level of a selected population

(in phenotypic standard deviation units), $H_{(g)}$, as a function of the number of generations $(g)$.

$M$ is for mass selection and $C$ is for combined selection.

After the letter is the value of $B$ (proportion of males from selected colonies).

Other parameters have the following values:

$$
\mathrm{R}=10, \mathrm{~S}=12, \mathrm{~V}_{\mathrm{A}}^{\mathrm{q}}=\mathrm{V}_{\mathrm{A}}^{\mathrm{qW}}=\mathrm{V}_{\mathrm{D}}^{\mathrm{W}}=\mathrm{V}_{\mathrm{D}}^{\mathrm{W}}=0,025, \mathrm{~V}_{\mathrm{A}}^{\mathrm{W}}=\mathrm{V}_{\mathrm{D}}^{\mathrm{q}}=0,050
$$




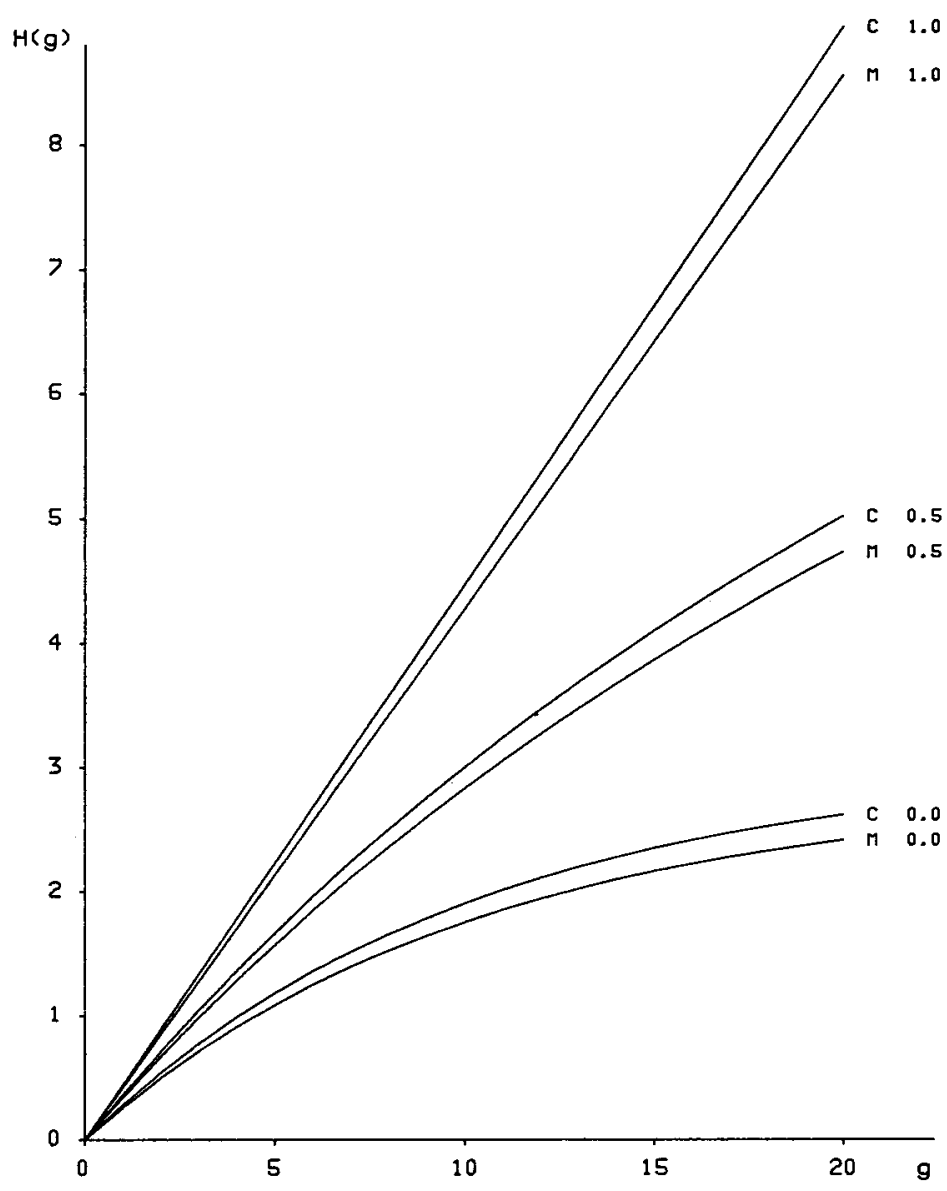

Fig. 2. - Evolution théorique du niveau génétique d'une population sélectionnée (unité : écart-type phénotypique) en fonction des générations.

Courbes $\mathrm{M}$ : sélection massale (indice JA).

Courbes $\mathrm{C}$ : sélection combinée (indice $\mathrm{JB}$ ).

La valeur indiquée après la lettre est celle du paramètre B (proportion de mâles en provenance des colonies sélectionnées).

Les autres paramètres ont les valeurs suivantes :

$$
\mathrm{R}=10, \mathrm{~S}=12, \mathrm{~V}_{\mathrm{A}}^{\mathrm{q}}=\mathrm{V}_{\mathrm{A}}^{\mathrm{w}}=\mathrm{V}_{\mathrm{D}}^{\mathrm{G}}=\mathrm{V}_{\mathrm{D}}^{\mathrm{w}}=0,075, \mathrm{~V}_{\mathrm{A}}^{\mathrm{qw}}=\mathrm{V}_{\mathrm{D}}^{\mathrm{qw}}=0,150
$$

Fig. 2. - Theoretical change of the genetic level of a selected population (in phenotypic standard deviation units), $H_{(g)}$, as a function of the number of generations ( $g$ ).

$M$ is for mass selection and $C$ is for combined selection.

After the letter is the value of $B$ (proportion of males from selected colonies).

Other parameters have the following values:

$$
\mathrm{R}=10, \mathrm{~S}=12, \mathrm{~V} \mathcal{X}=\mathrm{V}_{\mathrm{A}}^{\mathrm{w}}=\mathrm{V}_{\mathrm{D}}^{\mathrm{y}}=\mathrm{V}_{\mathrm{D}}^{\mathrm{w}}=0,075, \mathrm{~V}_{\mathrm{A}}^{\mathrm{qw}}=\mathrm{V}_{\mathrm{D}}^{\mathrm{w}}=\mathbf{w}=\mathbf{1 5 0}
$$


nul). Enfin, nous avons considéré un effectif total de 120 colonies, réparties en 10 familles de 12 colonies $(\mathrm{R}=10, \mathrm{~S}=12)$ et la sélection combinée 2 a été éliminée pour la clarté de la représentation.

L'influence de l'ouverture de la population sélectionnée apparait nettement sur ces figures. En effet, avec ce modèle, une valeur importante de $\beta$ bénéficie à la population sélectionnée à la fois directement (davantage de mâles sélectionnés vont féconder les reines du plan) et indirectement (l'amélioration du niveau génétique de la population environnante est plus rapide et le niveau des mâles extérieurs est supérieur). Les courbes s'écartent de la linéarité lorsque les deux conditions suivantes sont réunies : variances génétiques élevées et population sélectionnée largement ouverte. Lorsque l'héritabilité est faible, le coefficient $\beta$ joue davantage sur la pente que sur la linéarité de la progression et l'avantage à la sélection combinée est plus important.

Reçu pour publication en octobre 1985. Accepté pour publication en mars 1987.

\section{SUMMARY}

\section{THEORETICAL STUDY OF THE SELECTION OF THE HONEY YIELD CHARACTER II. COMBINED SELECTION SCHEME OF HONEY BEE QUEENS IN NATURAL MATING}

This paper describes a selection scheme for beekeepers who need only have mastered queen rearing and who arc willing to improve the honcy production of their stock. Every generation, $S$ queens are reared from each of $\mathrm{R}$ selected colonies and are naturally mated with drones coming mainly from these sclected queens. The choice of these colonies is based on a sclection index which may take up to three kinds of information :

- the honey production values of the colony itsef,

- the values of colonies whose queens are the sisters of the queen of the colony (" sistercolonics $"$ ),

- the value of the " mother-colony ».

The response to selection involves both the "female" and the " male» pathways. With a fixed total number of colonies, this response is shown to be maximum when the number of selected colonies is minimum. However, a limiting value is imposed by the necessity of maintaining inbreeding below a given level. We have shown clscwhere (CHEvalet and Cornuet, $1982 \mathrm{~b}$ ) that with a value of 10 selected colonies per generation, the inbreeding mean coefficient was always kept under $10 \%$

The combined selection provides a relative increase of the response which may reach $68 \%$ compared to mass selection. The most favourable situation for combined selection is when the family size (S) is large and the genetic variances are low.

As mating is not controlled, there is an exchange of genes between the selected and surrounding populations. This results in a lower rate of improvement in the selected population but the selection benefits a larger popoulation. When the selected population is widely open to exterior drones and the genetic variances are high, the rate of improvement per generation slows down (Fig. 1 and 2 ). 


\section{ZUSAMMENFASSUNG}

\section{THEORETISCHE STUDIE ÜBER DAS MERKMAL « HONIGERTRAG » BEI DER HONIGBIENE II. SELEKTIONSSCHEMA VON BIENENKÖNIGINNEN IN NATÜRLICHER PAARUNG}

Diese Arbeit beschreibt ein Selektionsschema für Bienenzüchter, die zwar über Kenntnisse übcr dic Aufzucht von Bienenköniginnen verfügen, nun aber auch die Honigerzeugung ihrer Bienenvölker steigern möchten. In jeder Generation wird eine Zahl von S Königinnen von jedem der $R$ selektierten Völker aufgezogen und auf natürlichem Wege mit Drohnen gepaart, die vorwiegend von diesen selektionierten Königinnen abstammen. Die Auswahl dieser Völker beruht auf einem Selektionsindex, der auf folgenden Informationen beruht :

- Honigertrag des Volkes selbst,

- Honigertrag der Völker, deren Königinnen Schwestern der Königin des selektierten Volkes sind (« Schwester-Völker»),

- Honigertrag des « Muttervolkes".

Das Ergebnis der Selektion wird sowohl von der «weiblichen » wie der «männlichen " Abstammungslinie bestimmt. Es konnte gezeigt werden, daß dieses Ergebnis bei einer feststehenden Gesamtzahl von Völkern am höchsten ist, wenn die Zahl der selektierten Völker so niedrig als möglich gehalten wird (steigende "Schärfe" der Selektion). Durch die Notwendigkcit, die Inzucht unter einem gegebenen Niveau zu halten, wird jedoch eine Begrenzung dieser Selektionsschärfe erzwungen. Wir haben schon früher gezcigt (Chevalet und Cornuet, 1982 b), daß mit einer Zahl von 10 selektierten Völkern per Generation der mittlere Inzuchtkoeffizient ständig unter $10 \%$ gehalten werden kann.

Die kombinierte Selektion führt zu einer relativen Steigerung des Ergebnisses, die im Vergleich zur Masscnselektion $68 \%$ erreichen kann. Die günstigste Situation für eine kombinierte Selektion ist dann gegeben, wenn die Familiengröße (S) beträchtlich und die genetische Varianz klein ist.

Da die Paarung nicht kontrolliert wird, besteht ein Austauch von Genen zwischen der selektierten und den umgebenden Populationen. Das Ergebnis ist eine niedrigere Steigerungsrate in der selektierten Population, aber es profitiert eine größere Population von der Selektion. Ist dic selekticrte Population bei hoher genetischer Varianz weit geöffnet für dic umgebenden Drohnen, dann verlangsamt sich die Steigerungsrate per Generation.

\section{BIBLIOGRAPHIE}

Chevalet Cl., Cornuet J.M., 1982 a. - Etude théorique sur la sélection du caractère « production de miel » chez l'abeille. I. Modèle génétique et statistique. Apidologie, 13, 39-65.

Chevalet Cl., Cornuet J.M., 1982 b. - Evolution de la consanguinité dans une population d'abeilles. Apidologie, 13, 157-168.

Fresnaye J., Lavie P., Boesiger E., 1974. - La variabilité de la production de miel chez l'abeille de race noirc (Apis mellifica L.) et chez quelques hybrides interraciaux. Apidologie, 5, 1-120.

HiLl W.G., 1976. - Order statistics of correlated variables and implications in genetic selection programs. Biometrics, 32, 889-902.

Lush J.L., 1947. - Family merit and individual merit as bases for selection. Amer. Nat., 81, 241-261, 362-379.

Sales J., Hill W.G., 1976. - Effect of sampling errors on efficiency of selection indices. I. Use of information from relatives for single trait improvement. Anim. Prod., 22, 1-17.

Sмгтн М., 1969. - Optimum selection procedures in animal breeding. Animal Prod., 11, 433-442. 